\title{
Anatomical Variants of the Musculocutaneous Nerve- Report of Two Cases
}

\author{
Fernando Martinez ${ }^{1,2}$ and Guzmán Ripoll ${ }^{1 *}$ \\ ${ }^{1}$ Anatomy Department, Facultad de Medicina Universidad Claeh, Uruguay \\ ${ }^{2}$ Neurosurgery Department, Universidad de la República, Uruguay
}

*Corresponding author: Guzmán Ripoll, AnatomyDepartment, Facultad de Medicina Universidad Claeh, Maldonado, Uruguay, Email: guzman.ripoll@gmail.com

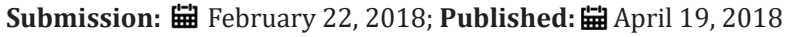

\begin{abstract}
Introduction: The muscle-cutaneous nerve $(\mathrm{MCN})$ is a mixed nerve, which is mainly responsible for shoulder flexion, elbow flexion and supination of the hand. The anatomical variations in this nerve are relatively frequent, fact with clinical and surgical implications.

Material and method: A retrospective review of 20 patients operated on with the Oberlin technique was made.

Results: Two variants were found in the 20 cases studied ( $10 \%$ of the cases): anastomosis between MCN and median nerve, and absence of the MCN.

Discussion: MCN variants are frequently described by the different authors. This has an embryological and phylogenetic explanation: the lateral and medial cords form the flexor plane of the muscles of the upper limb.

Conclusion: We present 2 cases: an unusual one of median nerve-MCN, and another absence of it. We emphasize that the knowledge of these variants have clinical, surgical and neuro-physiological applications.
\end{abstract}

\section{Introduction}

The musclecutaneous nerve (MCN) is a mixed nerve, which from the motor point of view is mainly responsible for shoulder flexion, elbow flexion and supination of the hand. This is done through the muscles that innervate: coracobrachial, biceps brachialis and anterior brachial [1-3]. Anatomically the MCN belongs to the flexor plane, along with the median and ulnar nerves. For this reason, anastomosis and variations in the motor innervations pattern of these nerves can appear during embryological development. The anatomical variations of the peripheral nerves are relatively frequent and their study has clinical and surgical importance, since they can vary the clinical presentation of some pathologies or even, they can lead to the erroneous interpretation of paraclinical studies, such as electro-diagnostic studies. In the present article two variants of the MCN were found in the course of brachial plexus repair surgeries are reported.

\section{Material and Method}

A retrospective review was made of 20 patients who underwent repair of the brachial plexus using the Oberlin technique or some of its modifications. The Oberlin technique consists in the reinnervation of the biceps brachial nerve with fascicles of the ulnar nerve (Figure 1). The modifications of this technique are basically the use of fascicles of the median nerve to reinforce the anterior brachial muscle (Figure 2). All selected patients had brachial plexus injuries with involvement of the upper trunk (C5C6) or upper and middle trunks (C5-C7). In all patients, the internal aspect of the arm was approached and anatomical recognition of the musculocutaneous, ulnar and median nerves. In addition, intraoperative neurophysiological testing was performed by stimulation with BEIC IGFA III Stim stimulator (BEIC, Rosario, Argentina). $1 \mathrm{~Hz}$ frequency and $1 \mathrm{~mA}$ intensity stimulation was used, increasing up to $5 \mathrm{~mA}$ in case of not obtaining the desired response. With this intensity, if there was no answer we interpreted it as a negative response.

\section{Results}

Two variants were found in the 20 cases studied ( $10 \%$ of cases). The first case was an anastomosis between the MCN and median nerves (MN). The patient had a traumatic brachial plexus injury that affected shoulder abduction and elbow flexion completely. He also had a partial involvement of: arm extension, extension and fist flexion and hand intrinsic. The clinical lesion path in this case 
was C5-C6 complete, C7-T1 partial. A superior upper arm approach was planned to perform the Oberlin technique, although given that the Flexor Carpi Ulnaris muscle nerve did not have good power, the possibility of neurotization of the Biceps Brachialis nerve with fascicles of the MN was proposed technique variant of Oberlin known as Oberlin II. The inner face of the upper third of the arm was approached and the musculocutaneous nerve was identified. The stimulation of this nerve had a negative response, as expected by the clinical examination. The Ulnar nerve had a weak response, so we discarded the Oberlin technique and proceeded to select a MN bundle.

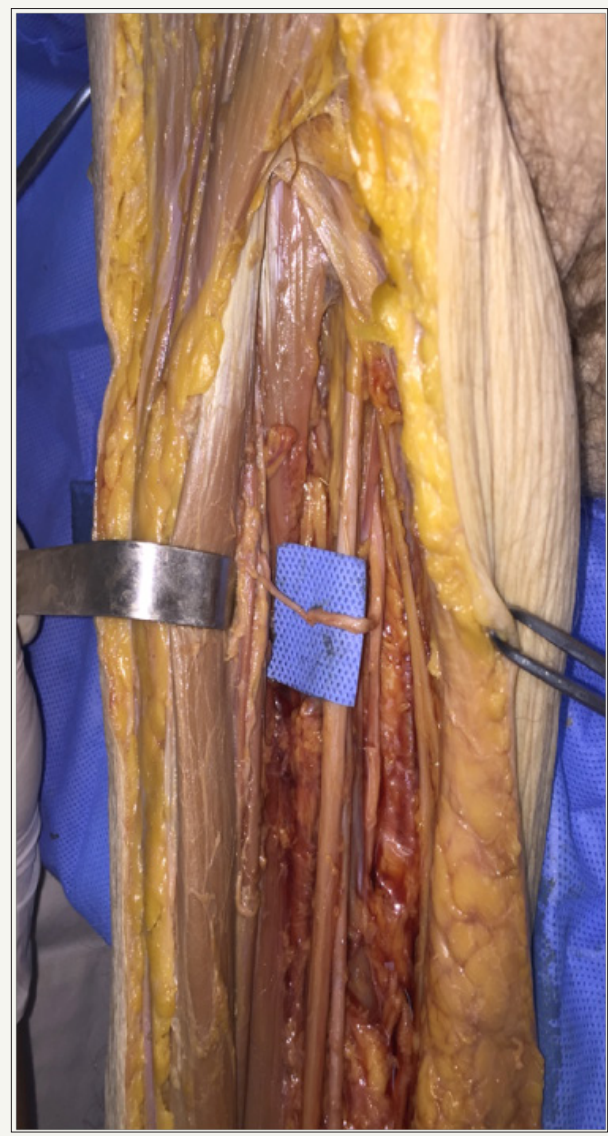

Figure 1: Anatomical dissection, nerve transfer of the ulnar nerve fascicle to the biceps brachialis nerve.

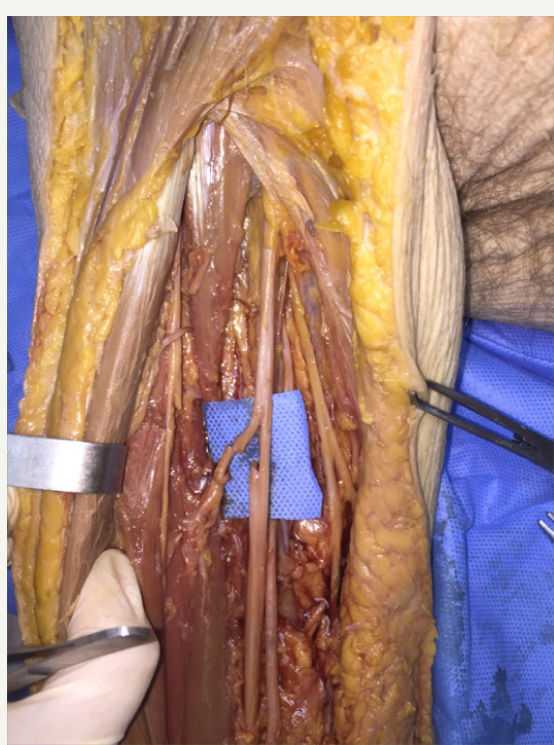

Figure 2: Anatomical dissection, transfer of the median nerve to the anterior brachial nerve. 
Given that in the repair of peripheral nerves it is ideal to make a suture without tension, the Biceps Brachialis nerve was dissected proximally, until its origin in the MCN. Then the epineural sheath was opened, performing a neurolysis to gain greater length of the biceps nerve, inside the musculocutaneous trunk. It was in this proximal dissection, in the trunk of the musculocutaneous nerve, that an anastomosis was identified between the MCN and the MN (Figure 3). The stimulation of the anastomosis and of the musculocutaneous nerve itself proximal to it generated motor response of flexion of the fingers. Therefore, the fibers that transited from the NMC to the median innervated the extrinsic flexor muscles of the fingers. After identifying the anastomosis and its function, it was protected and no longer dissected in its vicinity. The biceps nerve was selected from the distal section of the NMC, performing the rest of the procedure without incident. In the postoperative period, the patient did not show any impairment of motor function in flexor digitorum. We conclude then that the anastomosis carried motor information for finger flexors from the MCN to the MN.

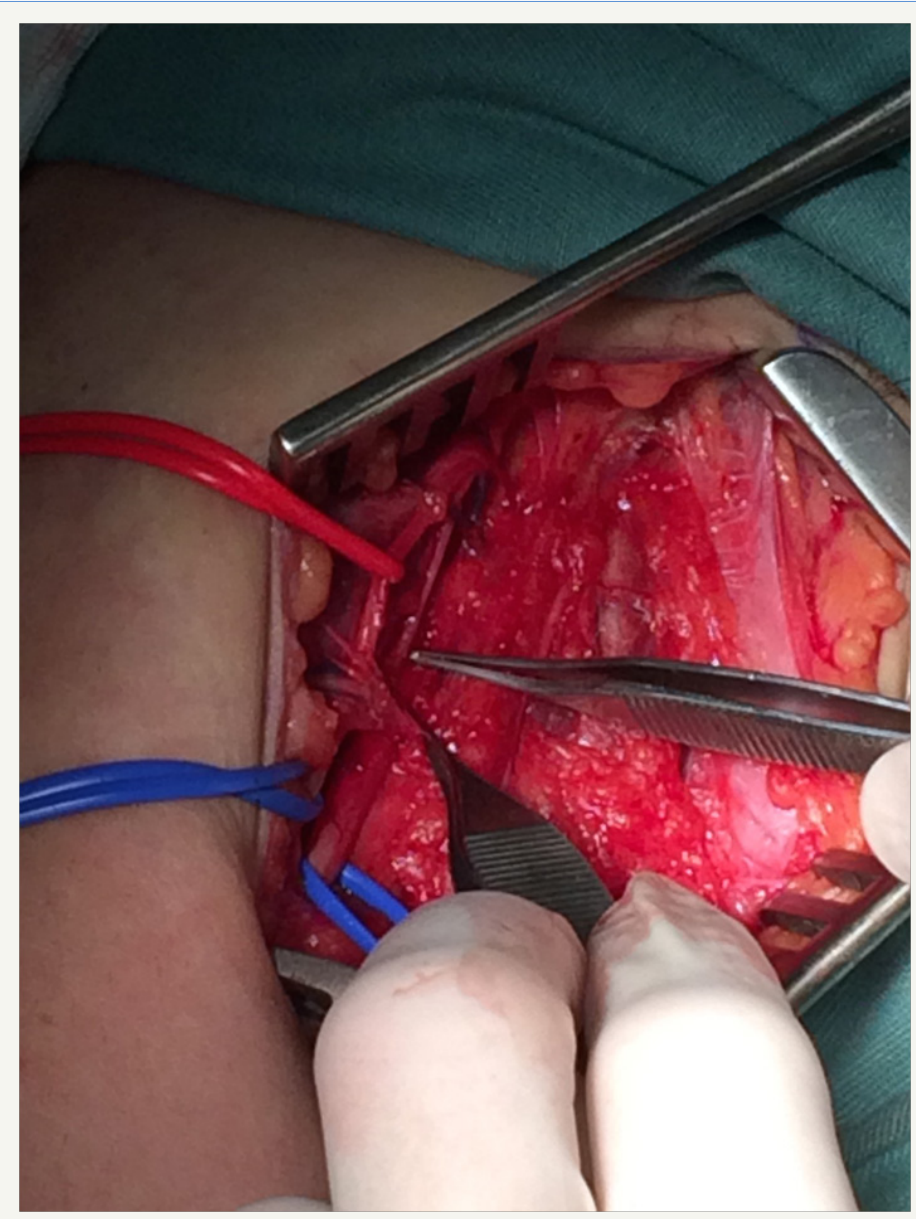

Figure 3: Intraoperative image muscle-cutaneous nerve anastomosis with median nerve.

The second case involved the absence of the musculocutaneous nerve. This variant was found in a 19-year-old patient with a complete traumatic lesion of the left brachial plexus. A plexus exploration and repair surgery was planned. In the supraclavicular exploration it was identified that the plexus was avulsed and retracted, so a neurotization of the phrenic nerve was planned to the biceps nerve. In the distal dissection, the musculocutaneous nerve was not identified. The motor branches for the biceps originated from the median nerve; it was not dissected further distally to see the distribution of the branches of the anterior brachial since it was not part of the surgical act. No neural structure similar to the musculocutaneous nerve was identified throughout the surgical field; the ulnar nerve was placed in its usual position.

\section{Discussion}

The MCN arises from the lateral cord of the brachial plexus and receives information from the roots $\mathrm{C} 5, \mathrm{C} 6$ and $\mathrm{C} 7$. The lateral root of the median nerve is also born from the lateral cord (3).

After its origin, the MCN descends in the arm across the coracobrachial muscle and is deep in relation to the biceps brachialis wichinnervates, goes laterally and gives the brachial anterior branch. It ends giving the sensory innervation of the skin of the lateral face of the forearm. MCN variants are described between 1.4 and $15 \%$ of cases [1,4-9] The most common variants are: origin from nerve trunks different to the lateral cord (posterior cord, medial cord, more than one root of origin), path without 
perforation of the coracobrachial muscle, absence of MCN or exchange of anastomosis with the median nerve [3].

The absence of MCN is unusual and the most frequent is that the muscles innervated by the MCN receive branches of the lateral cord directly, or of the median nerve [4,9-11]. This has an embryological and phylogenetic explanation: the lateral and medial cords form the flexor plane of the muscles of the upper limb. In some species of mammals there are only equivalents to the median and ulnar nerves and the branches for the flexor muscles of the arm are born from the median. Even in humans, the MCN is distinguished as a branch that is born from the middle one around 46-48 days of gestation [7]. When the MCN is absent, in general the coracobrachial muscle receives the innervation of the lateral cord, the lateral root of the median or the median. Biceps brachialis and anterior brachial muscles receive their branches from the median nerve [12]. In our case we could only observe the branches of the biceps being born from the median nerve and the distal part of the nerve could not be dissected because it is a surgical case. Likewise, the coracobrachial branch could not be observed. Anastomoses between peripheral nerves are known and relatively frequent. Some of these anastomoses have clear clinical implications, such as Martin-Gruber or Cannieu-Richet anasomosis, which occur between the ulnar and median nerves at the proximal and distal level with respect to the carpal tunnel (respectively).

The anastomoses between the median and musculocutaneous nerves are described between 2.8 and $63.5 \%$ of the cases, there being two possible variants according to their disposition: anastomosis from the MCN to the median or vice versa. Most often, the anastomosis is from the MCN to the median, which can be seen between 5 and $54.7 \%$ of cases and its most common topography is between the origin of the nerves of the biceps brachialis and the anterior brachial. Ballesteros and cols in an anatomical study on 106 cadaveric pieces found anastomosis in 21 cases $(19.8 \%)$. The most frequent topography was the one described above and represented $44.5 \%$ of cases with anastomosis. In our case, the anastomosis was found more proximal and represented $5 \%$ of the cases. This same topography is found by Ballesteros in about $2 \%$ of the pieces. It should be taken into account that our series is surgical and therefore, we did not systematically search for more distal anastomoses, having as anatomical repair the nerve of the biceps brachialis [13]. In addition, a fundamental fact must be emphasized: the figures for the incidence of anastomosis are due in their vast majority, to anatomical articles of cadaver dissections.

Therefore, it is difficult to be clear about the role and function of these anastomoses. Even the direction in which they are made, is based only on the direction of the nervous branch, without being able to check whether the information that transits through it is in one direction or another or even if it conducts motor or sensitive information. The fact of being able to observe an anastomosis and check its function with intra-operative neurophysiology, offers an invaluable opportunity to understand the significance of the exchange of information between peripheral nerves. In our case, the anatomical direction of the anastomosis was from the MCN to the median and from the functional point of view, we found that when the MCN was stimulated proximal to the anastomosis, there was contraction of the flexor muscles of the fingers. Therefore, the information that the anastomosis carried was in the same direction as the anatomical disposition of it. Although it is rare, the isolated lesion of the MCN (iatrogenic or traumatic) located proximal with respect to an anastomosis of this type, would have as a consequence the paresis of the flexion of the extrinsic muscles of the hand, associated with the plejia of the flexion Of elbow. This must be taken into account not only in the scenario of traumatic injuries of the MCN, but in its possible application in the anesthetic blocks of peripheral nerves [14]. Finally, given the patients who suffer a brachial plexus lesion may have variable distribution patterns, the intra-operative neurophysiological finding clearly leads us to the topographic approach.

\section{Conclusion}

We present a rare case of median nerve-MCN anastomosis, in which it was found that the information transited from the MCN to the median nerve. It had a clearly motor function, without being able to rule out its sensitive role due to intra-operative neurostimulation. The knowledge of this variant has clinical, surgical and neurophysiological applications.

\section{References}

1. Testut L (1921) Traité D’Anatomie Humaine. Tome premier. Octave Doin, Paris, France 1921: 943-945.

2. Testut L (1922) Traité D’Anatomie Humaine. Tome troisieme. Octave Doin, Paris, France 1922: 169-172.

3. Russell SM (2006) Examination of peripheral nerve injuries. An anatomical approach. Thieme, New York, USA.

4. Sarkar A, Saha A (2014) Bilateral absence of musculocutaneous nerve: a case report. J Clin Diagn Res 8(9): AD06-AD07.

5. Bhanu PS, Sankar KD (2012) Bilateral absence of musculocutaneous nerve with unusual branching pattern of lateral cord and median nerve of brachial plexus. Anat Cell Biol 45(3): 207-210.

6. Datta I, Ghoshal AK, Ray A (2011) Complete absence of lateral root of median nerve and communication of musculocutaneous nerve with median nerve, a case report. J Indian Med Assoc 109(5): 341-342.

7. Kaur P, Kumar R, Jain A (2014) Variations in innervation of muscles in anterior compartment of arm-a cadaveric study. J Clin Diagn Res 8(5): AC01-AC03.

8. Uzel AP, Bulla A, Steinmann G, LaurentJoye M, Caix P (2011) Absence of the musculocutaneous nerve and its distribution from median nerve: About two cases and literature review. Morphologie 95(311): 146-150.

9. Vollala VR, Nagabhooshana S, Bhat SM, Potu BK, Rodrigues V, et al. (2009) Multiple arterial, neural and muscular variations in upper limb of a single cadaver. Rom J Morphol Embryol 50(1): 129-135.

10. Song WC, Jung HS, Kim HJ, Shin C, Lee BY, et al. (2003) A variation of the musculocutaneous nerve absent. Yonsei Med J 44(6): 1110-1113.

11. Fregnani JH, Macéa MI, Pereira CS, Barros MD, Macéa JR (2008) Absence of the musculocutaneous nerve: a rare anatomical variation with possible clinical-surgical implications. Sao Paulo Med J 126(5): 288-290. 
12. Soubhagya N, Ashwin K, Madhan SJK, Latha VP, Vasudha S, et al. (2008) Four-headed biceps and triceps brachii muscles, with neurovascular variation. Anat SciInt 83(2): 107-111.

13. Ballesteros L, Forero P, Buitrago Edna (2014) Communication between the musculocutaneous and median nerves in the arm: an anatomical study and clinical implications. Rev Bras Ortop 50(5): 567-572.
14. Loukas M, Abel N, Tubbs RS, Matusz P, Zurada A, et al. (2011) Neural interconnections between the nerves of the upper limb and surgical implications. J Neurosurg 114(1): 225-235. (c) 1 BY $\begin{aligned} & \text { Creative Commons Attribution } 4.0 \\ & \text { International License }\end{aligned}$

For possible submissions Click Here

Submit Article

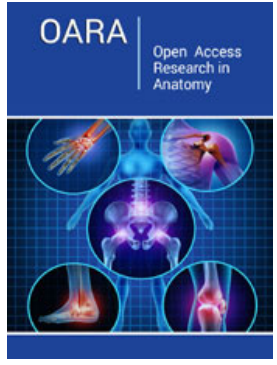

\section{Open Access Research in Anatomy}

\section{Benefits of Publishing with us}

- High-level peer review and editorial services

- Freely accessible online immediately upon publication

- Authors retain the copyright to their work

- Licensing it under a Creative Commons license

- Visibility through different online platforms 